Pacific Journal of Mathematic 


\title{
A QUASI-DECOMPOSABLE ABELIAN GROUP WITHOUT PROPER ISOMORPHIC QUOTIENT GROUPS AND PROPER ISOMORPHIC SUBGROUPS
}

\author{
JoHN M. IRWIN AND TAKASHI ITO
}

\begin{abstract}
All of the group in this paper are abelian $p$-groups without elements of infinite height. A group is said to be quasiindecomposable if whenever $H$ is a summand of $G$ then either $H$ or $G / H$ is finite. The $p$-socle of $G$ is the sub-group consisting of all the elements $x$ in $G$ such that $p x=0$.

In this paper it is shown that there are conditions that can be imposed on the socle of $G$ which are sufficient for $G$ to (a) have no proper isomorphic subgroups; (b) have no proper isomorphic quotient groups; and (c) be quasiindecomposable. Furthermore, it is shown that groups which make these results meaningful actually exist.
\end{abstract}

Let the cardinality of a group $G$ be either $\boldsymbol{\aleph}_{0}$ or greater than $c=2^{\mathbf{x}_{0}}$. Then, as is well known, $G$ has a proper isomorphic subgroup and a proper isomorphic quotient group. However P. Crawley [3] showed that the cardinality $c$ is exceptional. He gave an example $G_{0}$ of cardinality $c$ which has a standard basic subgroup and no proper isomorphic subgroups. After Crawley's example appeared, it was clear that a group, of cardinality $c$ and with a standard basic subgroup, supplies examples of groups with strange but interesting properties. In fact R. S. Pierce [7] gave an example $G_{1}$ which has no proper isomorphic subgroups and no proper isomorphic quotient groups. And he gave also in [7] an example $G_{2}$ which is quasi-indecomposable, that is, every direct summand $H$ of $G_{2}$ is either finite or $G_{2} / H$ is finite.

The relationship between the above three properties (no proper isomorphic subgroups, no proper isomorphic quotient groups and quasiindecomposability) of a group $G$ with the cardinality $c$ and a standard basic subgroup seems to authors an interesting problem. In this paper we shall give some results about this problem. In our approach the topological structure of the $p$-socle of the torsion completion of $G$ will be used in an essential way. Theorem 1 tells us that the situation of the $p$-socle of $G$ in the $p$-socle of the torsion completion of $G$ gives us sufficient conditions for these three properties of $G$. In some sense it shows a relationship between the three properties. Theorem 2 shows the existence of a group which has all three properties. Theorem 3 shows the existence of a group which has no proper isomorphic subgroups and no proper isomorphic quotient groups but which is quasi-decomposable. Now we want to add a simple proof of the following fact which 
was mentioned in the opening of this section.

Let $G$ be an infinite reduced p-group with card $G=\boldsymbol{\aleph}_{0}$ or card $G>c$. Then $G$ has a proper isomorphic subgroup and a proper isomorphic quotient group.

Proof. For simplicity we divide the proof into

Case 1; Suppose $G$ is bounded. Then $G=\sum_{k=1}^{n} B_{k}$ where $B_{k}$ is a direct sum of cyclic groups of order $p^{k}, B_{k}=\sum C\left(p^{k}\right)$. Now clearly one of these $B_{k}$ 's is infinite and throwing out a cyclic summand of $B_{k}$ yields the desired subgroup and quotient group.

Case 2. Suppose card $G=\boldsymbol{\aleph}_{0}$ and $G$ is unbounded. Then $G=$ $H \oplus K$ where $H$ is an unbounded direct sum of cyclic groups (Exercise 19 (a), p. 143 in [4]). It is easy to find a proper subgroup $A$ of $H$ which is isomorphic to $H$ and a non-zero subgroup $B$ of $H$ such that $H / B \cong H$. Whence we obtain our proper isomorphic subgroup $A \oplus K$ and our proper isomorphic quotient group $G / B$.

Case 3. Suppose $G$ is unbounded with card $G>c$, and $B=\sum_{k=1}^{\infty} B_{k}$ is a basic subgroup where $B_{k}=\sum C\left(p^{k}\right)$. Then $G=B_{1} \oplus B_{2} \oplus \cdots \oplus$ $B_{n} \oplus G_{n}$ for all $n$ (Theorem 29.3 in [4]). But as is well known (card $B)^{\aleph_{0}} \geqq \operatorname{card} G>c$ so that some $B_{n}$ must be infinite. Now throwing out a cyclic summand of $B_{n}$ yields the result as in Case 1 and the proof is complete.

2. Sufficient conditions for the three properties. Let $p>1$ be a fixed prime number, $C\left(p^{n}\right)$ be a cyclic group of order $p^{n}, \Sigma$ be the direct sum of cyclic groups $C\left(p^{n}\right)$, II be the direct product of cyclic groups $C\left(p^{n}\right)$ and $C$ be the torsion group of $\Pi$, that is, $\Sigma$ is the standard basic group and $C$ is the torsion completion of $\Sigma$.

The $p$-socle $C[p]$ of $C$ is a vector space over the prime field of characteristic $p$ and can be topologized as a totally disconnected compact topological group, because $\Pi$ is clearly a totally disconnected compact topological group with respect to the product topology of compact discrete topologies and the $p$-socle $C[p]$ of $C$ is the closed subgroup $\{x \mid x \in \Pi, p x=0\}$ of $\Pi$. Actually $U_{n}=\{x \mid x \in C[p]$ and $h(x) \geqq n\}=\left(p^{n} C\right)[p](n=1,2 \cdots)$ are open compact subgroups of $C[p]$ and $\left\{U_{n}\right\}$ is a fundamental system of 0 -neighborhoods in $C[p]$. These two structures on $C[p]$ which are a vector space and a totally disconnected compact group are used in an essential way in this paper.

Every continuous group homomorphism $T$ on $C[p]$ defines compact subgroups $E_{q}(T)=\{x \mid x \in C[p]$ and $T x=q x\}(0 \leqq q<p)$ and the compact subgroup $E(T)=E_{0}(T) \oplus E_{1}(T) \oplus \cdots \oplus E_{p-1}(T)$. We can define naturally two types of continuous group homomorphism on $C[p]$ as follows. $T$ is a singular homomorphism if $E(T)$ is an open compact subgroup of $C[p]$. For instance a continuous projection on $C[p]$ is 
singular. $T$ is a strongly singular homomorphism if for some $q E_{q}(T)$ is an open compact subgroup. If a continuous group homomorphism $T$ on $C[p]$ has a dense subgroup which is invariant under $T$ and on which $T$ is one to one, $T$ is called a semi-isomorphism on $C[p]$.

We have the following theorem which is fundamental to the ideas in what follows.

THEOREM 1. Let $G$ be a pure subgroup of $C$ which contains $\Sigma$ and $G[p]$ be the p-socle of $G$.

(1) If $G[p]$ is not invariant under any nonsingular onto homomorphism on $C[p]$, then $G$ has no proper isomorphic quotient groups.

(2) If $G[p]$ is not invariant under any nonsingular semiisomorphism on $C[p]$, then $G$ has no proper isomorphic subgroups.

(3) If $G[p]$ is not invariant under any nonstrongly singular projection on $C[p]$, then $G$ is quasi-indecomposable.

Proof. Suppose $\phi$ is a homomorphism of $G$ into $G$. The purity of $G$ in $C$ implies $\varphi\left(G[p] \cap U_{n}\right) \subset U_{n}$ for all $n=1,2, \cdots$. This means that the restriction of $\varphi$ to $G[p]$ is continuous on $G[p]$. since $G[p] \supset \Sigma[p]$ and $\Sigma[p]$ is dense in $C[p],\left.\varphi\right|_{G[p]}$ has a unique continuous homomorphism extension $T$ on $C[p]$. Clearly $G[p]$ is invariant under $T$ and $T\left(U_{n}\right) \subset U_{n}$ for all $n=1,2, \cdots$. If this $T$ is singular, then there exists a positive integer $N$ such that

$$
T\left(U_{N}\right) \subset U_{N} \subset E(T) .
$$

Then we have the following decomposition of $G[p]$,

$$
\begin{aligned}
G[p] & =\left(G[p] \cap U_{N}\right) \oplus R_{N}=\left(E_{0}(T) \cap G[p] \cap U_{N}\right) \\
& \oplus\left(E_{1}(T) \cap G[p] \cap U_{N}\right) \oplus \cdots \oplus\left(E_{p-1}(T) \cap G[p] \cap U_{N}\right) \oplus R_{N},
\end{aligned}
$$

where $R_{N}$ is a finite subgroup of $G[p]$.

Because $C[p] / U_{N}$ is finite and $G[p] / G[p] \cap U_{N}$ is isomorphic to a subgroup $C[p] / U_{N}$, so the dimension of $G[p] / G[p] \cap U_{N}$ as a vector space over the prime field of characteristic $p$ is finite. Hence there exists a finite subgroup $R_{N}$ of $G[p]$ such that $G[p]=\left(G[p] \cap U_{N}\right) \oplus R_{N}$. The decomposition of $G[p] \cap U_{N}$ can be shown as follows. For each $x$ in $G[p] \cap U_{N} x$ is the sum of $z_{q} \in E_{q}(T)(0 \leqq q<p) ; x=\sum_{1=0}^{p-1} z_{q}$. Then we have $\varphi^{\nu}(x)=\sum_{q=0}^{p-1} T^{\nu} z_{q}=\sum_{q=0}^{p-1} q^{\nu} z_{q}$ for $0 \leqq \nu \leqq p-1$. Since the determinant of Vandermonde's matrix is not zero $\bmod p$, each $z_{q}$ $(0 \leqq q \leqq p-1)$ is a linear combination of $x, \varphi(x), \cdots, \varphi^{p-1}(x)$. This means $z_{q} \in E_{q}(T) \cap G[p] \cap U_{N}$ for $0 \leqq q \leqq p-1$.

Proof of (1). Suppose $\varphi$ is an onto homomorphism of $G$. Then 
the continuous extension $T$ of $\left.\varphi\right|_{G[p]}$ is clearly an onto homomorphism of $C[p]$ and $G[p]$ is invariant under $T$. By our assumption $T$ must be singular, so we have the above decomposition of $G[p]$. Put $Q_{N}=$ $\left(E_{1}(T) \cap G[p] \cap U_{N}\right) \oplus\left(E_{2}(T) \cap G[p] \cap U_{N}\right) \oplus \cdots \oplus\left(E_{p-1}(T) \cap G[p] \cap U_{N}\right)$, clearly $\varphi\left(Q_{N}\right)=Q_{N}$ and $\varphi$ is an isomorphism on $Q_{N}$, and

$$
\left(E_{0}(T) \cap G[p] \cap U_{N}\right) \oplus R_{N} \cong G[p] / Q_{N}=\varphi(G[p]) / \varphi\left(Q_{N}\right) \cong \varphi\left(R_{N}\right)
$$

but $\operatorname{dim} \varphi\left(R_{N}\right) \leqq \operatorname{dim} R_{N}<+\infty$. This implies that $E_{0}(T) \cap G[p] \cap U_{N}=$ $\{0\}$ and $R_{N}$ is isomorphic to $\varphi\left(R_{N}\right)$ by $\varphi$. Therefore $\left.\varphi\right|_{G[p]}$ is an isomorphism on $G[p]$. Let $0 \neq x \in G$ and the order of $x=p^{n}>1$, then $0 \neq \varphi\left(p^{n-1} x\right)=p^{n-1} \varphi(x)$, so $\varphi(x) \neq 0$. Thus $\varphi$ must be an isomorphism on $G$.

Proof of (2). Suppose $\varphi$ is an isomorphism of $G$ into $G$. We have to show $\varphi(G)=G$. The continuous extension $T$ of $\left.\varphi\right|_{G[p]}$ is a semiisomorphism and $G[p]$ is invariant under $T$. By our assumption $T$ must be singular, so we have the same decomposition of $G[p]$ as above. First of all we can see $\varphi(G[p])=G[p]$. Automatically

$$
E_{0}(T) \cap G[p] \cap U_{N}=\{0\},
$$

because $\varphi$ is one to one, therefore $G[p]=Q_{N} \oplus R_{N} \cong \varphi\left(Q_{N}\right) \oplus \varphi\left(R_{N}\right)=$ $Q_{N} \oplus \varphi\left(R_{N}\right) \subset G[p]$ but $\operatorname{dim} R_{N}=\operatorname{dim} \varphi\left(R_{N}\right)<+\infty$, this implies $\varphi(G[p])=$ $G[p]$. Next we can see $\varphi(G) \supset G\left[p^{2}\right]$. The group $H=\{x \mid x \in G$ and the first $N-1$ coordinates in $I I$ are zero $\}$ is a direct summand of $G$ and

$$
\begin{aligned}
H[p] & =G[p] \cap U_{N}=Q_{N} \\
& =\left(E_{1}(T) \cap Q_{N}\right) \oplus\left(E_{2}(T) \cap Q_{N}\right) \oplus \cdots \oplus\left(E_{p-1}(T) \cap Q_{N}\right) .
\end{aligned}
$$

We can take a finite group $L$ such that $G=H \oplus L$. We have to show first $\varphi(G) \supset H\left[p^{2}\right]$. For arbitrary $x$ in $H\left[p^{2}\right] p x=\sum_{q=0}^{p-1} z_{q}$ for some $z_{q} \in E_{q}(T) \cap Q_{N}(1 \leqq q \leqq p-1)$, then each $z_{q}$ is a linear combination of $p \varphi(x), p \varphi^{2}(x), \cdots, p \varphi^{p-1}(x)$. This means that there exist $x_{q} \in G$ $(1 \leqq q \leqq p-1)$ such that $z_{q}=p \varphi\left(x_{q}\right)$ for $1 \leqq q \leqq p-1$. Therefore $p x=\sum_{q=1}^{p-1} p \varphi\left(x_{q}\right)$, so $x-\varphi\left(\sum_{q=1}^{p-1} x_{q}\right) \in G[p]$, but $G[p]=\varphi(G[p])$ implies $x \in \varphi(G)$. Now $\varphi(G) \supset G\left[p^{2}\right]$ can be shown. For $x \in G\left[p^{2}\right]$ there exists a positive integer $M$ and integers $r_{i}, 0 \leqq r_{i} \leqq p-1$ (at least one of them is not zero) such that $\sum_{i=0}^{M} r_{i} p \varphi^{i}(x) \in Q_{N}=H[p]$, because $G[p] / Q_{N}$ is finite dimensional. Since $\varphi\left(Q_{N}\right)=Q_{N}$, we can assume $r_{0}=1$ without loss of generality. Then we find $z \in H\left[p^{2}\right]$ such that $p \sum_{i=0}^{M I} r_{i} \varphi^{i}(x)=p \boldsymbol{z}$. But $H\left[p^{2}\right] \subset \varphi(G)$ has been shown, so $z=\varphi\left(z^{\prime}\right)$ for some $z^{\prime} \in G$, therefore $x+\sum_{i=1}^{M} r_{i} \varphi^{i}(x)-\varphi\left(z^{\prime}\right) \in G[p]=\varphi(G[p])$, this implies $x \in \varphi(G)$. Now we can see $\varphi(G) \supset G\left[p^{n}\right]$ for all $n=1,2 \ldots$ by induction. Namely in general $\varphi(G) \supset G\left[p^{n}\right]$ and the special form of $\varphi$ on $Q_{N}$ imply $\varphi(G) \supset H\left[p^{n+1}\right]$. And $\varphi(G) \supset H\left[p^{n+1}\right]$ and the finiteness of $L$ imply $\varphi(G) \supset G\left[p^{n+1}\right]$. 
Proof of (3). Suppose $G$ is the direct sum of two subgroups $G_{1}$ and $G_{2}$ and $\varphi$ is the projection onto $G_{1}$. The continuous extension $T$ of $\left.\varphi\right|_{G[p]}$ is also a projection defined on $C[p]$, therefore $C[p]=E_{0}(T) \oplus E_{1}(T)$ and $G[p]=\left(E_{0}(T) \cap G[p]\right) \oplus\left(E_{1}(T) \cap G[p]\right)$. Since $G[p]$ is invariant under $T, T$ must be strongly singular by our assumption about $G[p]$. Suppose $E_{1}(T)$ is open, then $E_{0}(T)$ is finite, hence $G_{2}[p]=E_{0}(T) \cap G[p]$ is finite. The finiteness of $G_{2}[p]$ implies the finiteness of $G_{2}$.

The following is a direct corollary of Theorem 1 .

CoROLlary. Let $G$ be a pure subgroup of $C$ which contains $\Sigma$. If $G[p]$ is not invariant under any nonstrongly singular homomorphism on $C[p]$, then $G$ has the three properties stated in (1), (2) and (3) in Theorem 1. Namely $G$ has no proper isomorphic quotient group and no proper isomorphic subgroup, and $G$ is quasi-indecomposable.

\section{Existence theorem}

THEOREM 2. There exists a pure subgroup $G$ of $C$ which contains $\Sigma$ and satisfies three properties;

(1) $G$ has no proper isomorphic quotient groups,

(2) $G$ nas no proper isomorphic subgroups,

(3) $G$ is quasi-indecomposable.

And an arbitrary pure subgroup $H$ of $C$ such that $H$ contains $\Sigma$ and $H[p]=G[p]$ satisfies above three properties.

This theorem comes from the corollary of Theorem 1 and following two lemmas. Lemma 1 is known as the purification property, so we omit the proof of Lemma 1 (see more general form in [6]).

LeMma 1. For an arbitrary subgroup $Q$ between $\Sigma[p]$ and $C[p]$ there exists a pure subgroup $G$ of $C$ such that $G$ contains $\Sigma$ and $G[p]=Q$.

Lemma 2. For any family $\left\{T_{\lambda} \mid \lambda \in \Lambda\right\}$ of nonstrongly singular homomorphisms on $C[p]$ there exists a subgroup $Q$ between $\Sigma[p]$ and $C[p]$ such that $Q$ is not invariant under any $T_{\lambda}(\lambda \in \Lambda)$.

The existence of such $Q$ can be shown by transfinite induction which is Crawley's idea in [3]. We need following lemma which is also essentially Crawley's.

LEMMA 3. Suppose $T$ is a nonstrongly singular homomorphism on $C[p]$. Then there exists a one-parameter family $\Delta(T)=\left\{x_{t} \mid 0 \leqq t \leqq 1\right\}$ of elements in $C[p]$ such that four elements $x_{s}, x_{t}, T x_{s}$ and $T x_{t}$ are 
linearly independent for arbitrary $s \neq t$.

Proof. The proof can be divided into two cases $(a)$ and $(b)$.

(a) $T$ is singular but not strongly singular. In this case, by Baire's category theorem $(C[p]$ is a complete metric space) there are at least two $q$ and $q^{\prime}$ such that both $E_{q}(T)$ and $E_{q^{\prime}}(T)$ are infinite compact groups, so card $E_{q}(T)=$ card $E_{q^{\prime}}(T)=c$ (for instance, see [5], p. 31). Therefore $\operatorname{dim} E_{q}(T)=\operatorname{dim} E_{q^{\prime}}(T)=c$. Let $\left\{y_{t} \mid 0 \leqq t \leqq 1\right\}$ be a basis of $E_{q}(T)$ and $\left\{y_{t}^{\prime} \mid 0 \leqq t \leqq 1\right\}$ be a basis of $E_{q^{\prime}}(T)$. Then $\Delta(T)=\left\{y_{t}+y_{t}^{\prime} \mid 0 \leqq t \leqq 1\right\}$ is the desired family.

(b) $T$ is not singular. In this case, by Baire's category theorem $U_{n} / E(T) \cap U_{n}$ are infinite compact groups for all $n=1,2 \cdots$, so as above $\operatorname{dim} U_{n} / E(T) \cap U_{n}=c$. Hence $U_{n}=\left(E(T) \cap U_{n}\right) \oplus D_{n}$ with $\operatorname{dim}$ $D_{n}=c$ for all $n=1,2, \cdots$. Take $0 \neq x_{0} \in D_{1}$, then $x_{0}$ and $T x_{0}$ are linearly independent. Let $\left\{z_{0}, z_{1}, \cdots, z_{p^{2}-1}\right\}$ be the group generated by $x_{0}$ and $T x_{0}$, then by the continuity of $T$ we can find $U_{M}$ such that $z_{i}+U_{M}+T\left(U_{M}\right)\left(0 \leqq i \leqq p^{2}-1\right)$ are mutually disjoint. For this $M$ we take a basis $\left\{y_{t} \mid 0 \leqq t \leqq 1\right\}$ of $D_{M}$. Then $\Delta(T)=\left\{x_{0}+y_{t} \mid 0 \leqq t \leqq 1\right\}$ is the desired system. Because, suppose $n_{1}\left(x_{0}+y_{t}\right)+n_{2}\left(T x_{0}+T y_{t}\right)=$ $n_{1}^{\prime}\left(x_{0}+y_{s}\right)+n_{2}^{\prime}\left(T x_{0}+T y_{s}\right)$ for $s \neq t$ where $n_{1}, n_{2}, n_{1}^{\prime}$ and $n_{2}^{\prime}$ are integers, then $n_{1} x_{0}+n_{2} T x_{0}+n_{1} y_{t}+n_{2} T y_{t}=n_{1}^{\prime} x_{0}+n_{2}^{\prime} T x_{0}+n_{1}^{\prime} y_{s}+n_{2}^{\prime} T y_{s}$, and $n_{1} x_{0}+n_{2} T x_{0}$ must be some $z_{i}$ and also $n_{1}^{\prime} x_{0}+n_{2}^{\prime} T x_{0}$ must be some $z_{j}$, but $z_{i}=z_{j}$ by our choice of $U_{M}$. This implies $n_{1}=n_{1}^{\prime} \bmod p$ and $n_{2}=n_{2}^{\prime} \bmod p$, therefore we have $n_{1} y_{t}+n_{2} T y_{t}=n_{1} y_{s}+n_{2} T y_{s}$, whence $n_{1}\left(y_{t}-y_{s}\right)=-n_{2} T\left(y_{t}-y_{s}\right)$. However $0 \neq y_{t}-y_{s} \in D_{M}$ and $D_{M} \cap E(T)=$ $\{0\}$, hence $n_{1}=n_{2}=0 \bmod p$.

Proof of Lemma 2. $\left\{T_{\lambda} \mid \lambda \in \Lambda\right\}$ is given, then card $\Lambda$ is at most $c$ (note that the cardinality of the set of all continuous homomorphisms on $C[p]$ is at most $c$, because $C[p]$ is a separable compact group). We assume that $\Lambda$ is a well ordered set of ordinal numbers which are less than $\Omega$, where $\Omega$ is the first ordinal number whose cardinality is $c$. Choose $e \in C[p]$ but $e \notin \Sigma[p]$, then we can construct a family of subgroups $R_{\lambda}(\lambda \in \Lambda)$ by transfinite induction as follows:

(a) $\Sigma[p]=R_{0} \subset R_{\lambda} \subset R_{\mu} \subset C[p]$ if $0 \leqq \lambda<\mu(\lambda, \mu \in \Lambda)$,

(b) card $R_{\lambda} \leqq$ card $\lambda \cdot \boldsymbol{\aleph}_{0}<c$ for all $\lambda \in \Lambda$,

(c) $e \notin R_{\lambda}$ but there exists $x_{\lambda} \in R_{\lambda} \cap \Delta\left(T_{\lambda}\right)$ such that $e-T_{\lambda} x_{\lambda} \in R_{\lambda}$. Suppose $R_{\lambda}$ has been constructed for all $\lambda<\mu \in \Lambda$. Let $R_{\mu}^{\prime}=\bigcup_{i<\mu} R_{\lambda}$. Then card $\left([e]+R_{\mu}^{\prime}\right) \leqq \operatorname{card} \mu \cdot \mathbf{Y}_{\mathbf{A}_{0}}<c$, where [e] is the group generated by $e$. The property of $\Delta\left(T_{\mu}\right)$ in Lemma 3 guarantees the existence of $x_{t 0} \in \Delta\left(T_{\mu}\right)$ such that $\left([e]+R_{\mu}^{\prime}\right) \cap\left(\left[x_{t 0}\right]+\left[T_{\mu} x_{t 0}\right]\right)=\{0\}$. Then $R_{\mu}=$ $R_{\mu}^{\prime}+\left[x_{t 0}\right]+\left[e-T_{\mu} x_{t 0}\right]$ is the desired subgroup. Let $Q=\bigcup_{\lambda \in A} R_{\lambda}$, then by (a) $Q$ is a subgroup of $C[p]$ which contains $\Sigma[p]$ and by (c) $Q$ is not invariant under any $T_{\lambda}(\lambda \in \Lambda)$. 
4. A quasi-decomposable group without proper isomorphic quotient groups and proper isomorphic subgroups.

THEOREM 3. There exists a pure subgroup $G$ of $C$ which contains $\Sigma$ and satisfies properties;

(1) $G$ has no proper isomorphic quotient groups,

(2) $G$ has no proper isomorphic subgroups,

(3) $G$ has a decomposition $G_{1} \oplus G_{2}$ such that $G_{1}$ and $G_{2}$ are not bounded.

The following lemma is essential for our proof of this theorem.

LEMMA 4. For any family $\left\{T_{\lambda} \mid \lambda \in \Lambda\right\}$ of nonsingular homomorphisms on $C[p]$ there exists a subgroup $Q$ between $\Sigma[p]$ and $C[p]$ such that $Q$ is not invariant under any $T_{2}(\lambda \in \Lambda)$ but invariant under the canonical projection $P_{e}$ onto even coordinates.

The outline of the proof of this lemma will be given later.

Proof of Theorem 3. Every element of $C$ has countable coordinates as an element of the product space $\prod_{n=1}^{\infty} C\left(p^{n}\right) ; x \in C$ is called an even (odd) element if all odd (even) coordinates are zero. For a subset $A$ of $C A^{e}\left(A^{0}\right)$ means the set of all even (odd) elements in $A$. Then clearly $C=C^{e} \oplus C^{0}$ and $\Sigma=\Sigma^{e} \oplus \Sigma^{0}$. By Lemma 4 there exists a subgroup $Q$ between $\Sigma[p]$ and $C[p]$ such that $Q$ is not invariant under any nonsingular homomorphisms on $C[p]$ but is invariant under $P_{e}$, therefore $\Sigma^{e}[p]=\Sigma[p]^{e} \subset Q^{e} \subset C[p]^{e}=C^{e}[p], \Sigma^{0}[p]=\Sigma[p]^{0} \subset Q^{0} \subset C[p]^{0}=$ $C^{0}[p]$ and $Q=Q^{e} \oplus Q^{0}$. With exactly the same proof as that of Lemma 1 we can show that there exists a pure subgroup $G_{1}\left(G_{2}\right)$ of $C^{e}\left(C^{0}\right)$ which contains $\Sigma^{e}\left(\Sigma^{0}\right)$ and $G_{1}[p]=Q^{e}\left(G_{2}[p]=Q^{0}\right)$. Clearly $G_{1}$ and $G_{2}$ are not bounded. Let $G=G_{1} \oplus G_{2}$, then $G$ is a pure subgroup of $C$ which contains $\Sigma$ and $G[p]=G_{1}[p] \oplus G_{2}[p]=Q^{e} \oplus Q^{0}=Q$. By Theorem $1 G$ has the properties (1) and (2) in Theorem 3.

The outline of the proof of Lemma 4. In order to prove Lemma 4 we can apply a similar method to the construction of $Q$ in Lemma 2. However before doing it we have to prepare some reformation of Lemma 3. Precisely our reformation is as follows, hereafter we shall use notations $A^{e}=P_{e}(A)\left(A^{0}=\left(I-P_{e}\right)(A)\right)$ for a subset $A$ of $C[p]$ and $x^{e}=P_{e} x\left(x^{0}=x-P_{e} x\right)$ for an element $x$ in $C[p]$.

For an arbitrary nonsingular homomorphism $T$ we can find a one-parameter family $\Delta(T)=\left\{x_{t} \mid 0 \leqq t \leqq 1\right\}$ of elements in $C[p]$ which has one of the following six properties; $1^{0}, 2^{0}, 3^{0}, 1^{e}, 2^{e}$ and $3^{e}$, 
$1^{0} x_{t}, T x_{t} \in C[p]^{0}$ for all $0 \leqq t \leqq 1$ and four elements $x_{s}, x_{t}, T x_{s}$ and $T x_{t}$ are linearly independent for arbitrary $s \neq t$,

$2^{0}$ there exists $q, 0 \leqq q \leqq p-1$ such that $x_{t} \in C[p]^{0}$ and

$$
T x_{t}-q x_{t} \in C[p]^{e}
$$

for all $0 \leqq t \leqq 1$ and four elements $x_{s}, x_{t}, T x_{s}-q x_{s}$ and $T x_{t}-q x_{t}$ are linearly independent for arbitrary $s \neq t$,

$3^{0} \quad x_{t} \in C[p]^{0}$ for all $0 \leqq t \leqq 1$ and six elements $x_{s}, x_{t},\left(T x_{s}\right)^{0},\left(T x_{s}\right)^{e}$, $\left(T x_{t}\right)^{0}$ and $\left(T x_{t}\right)^{e}$ are linearly independent for arbitrary $s \neq t$.

$1^{e}, 2^{e}$ and $3^{e}$ are dual properties $1^{0}, 2^{0}$ and $3^{0}$ by exchanging odd for even.

In the proof of this we have some difficulty coming from noncommutativity of nonsingular homomorphism and $P_{e}$. The proof in our original manuscript needs a long computation, in this paper we omit our detailed computation according to referee's suggestion but authors can supply the detailed proof to interested readers.

Using above $\Delta(T)$ the existence of $Q$ in Lemma 4 can be shown as follows. Let $\left\{T_{\lambda} \mid \lambda \in \Lambda\right\}$ be a given family of nonsingular homomorphisms on $C[p]$. We assume that $\Lambda$ is a well ordered set of ordinal numbers which are less than the first ordinal number whose cardinality is $c$. Choose $c \in C[p]$ but $c^{0}, c^{e} \notin \Sigma[p]$. By transfinite induction we can construct the following family of subgroups $R_{\lambda}(\lambda \in \Lambda)$;

(a) $\Sigma[p]=R_{0} \subset R_{\lambda} \subset R_{\mu} \subset C[p]$ if $0 \leqq \lambda<\mu(\lambda, \mu \in A)$,

(b) $\operatorname{card} R_{\lambda} \leqq \operatorname{card} \lambda \cdot \boldsymbol{\aleph}_{0}<c$ for all $\lambda \in \Lambda$,

(c) $R_{\lambda}$ is invariant under $P_{e}$ for all $\lambda \in \Lambda$,

(d) $c^{0}$ and $c^{e} \notin R_{\lambda}$ but there exists $x_{\lambda} \in R_{\lambda} \cap \Delta\left(T_{\lambda}\right)$ such that $c^{0}-T_{\lambda} x_{\lambda}$ or $c^{e}-T_{\lambda} x_{\lambda}$ or $c-T_{\lambda} x_{\lambda} \in R_{\lambda}$ for all $\lambda \in \Lambda$.

Suppose $R_{\lambda}$ has been constructed for all $\lambda<\mu \in \Lambda$. Let $R_{\mu}^{\prime}=$ $\bigcup_{\lambda<\mu} R_{\lambda}$. Then card $R_{\lambda}^{\prime} \leqq \operatorname{card} \lambda \cdot \boldsymbol{\aleph}_{0}<c$ and $R_{\lambda}^{\prime}$ is invariant under $P_{e}$ and $c^{0}$ and $c^{e} \notin R_{\lambda}^{\prime}$. Let $A\left(T_{k}\right)$ be one having one of properties $1^{0} \sim 3^{0}$ and $1^{e} \sim 3^{e}$. Suppose $\Delta\left(T_{\mu}\right)$ has property $1^{\circ}$, then we can find $x_{\mu} \in \Delta\left(T_{\mu}\right)$ such that $\left(R_{\mu^{\prime}}^{\prime}+\left[c^{0}\right]+\left[c^{e}\right]\right) \cap\left(\left[x_{\mu^{\prime}}\right] \oplus\left[T_{\mu} x_{\mu}\right]\right)=\{0\}$. Let

$$
R_{\mu}=R_{\mu}^{\prime}+\left[x_{\mu}\right]+\left[c^{0}-T_{\mu} x_{\mu}\right],
$$

then clearly $R_{\mu}$ satisfies above (a), (b) and (c). And $c^{0}$ and $c^{e} \in R_{\mu}$ also holds. Suppose $c^{0} \in R_{\mu}$, then $c^{0}=x+n x_{\mu}+m\left(c^{0}-T_{\mu} x_{\mu}\right)$ for some $x \in R_{\mu}^{\prime}$ and some integers $n$ and $m$, so $-x+(1-m) c^{0}=n x_{\mu}-m T_{\mu} x_{\mu}$, but by our choice of $x_{\mu}, n x_{\mu}-m T_{\mu} x_{\mu}=0$ and $x+(m-1) c^{0}=0$. This implies $n=m=0 \bmod p$ and $c^{0}=x \in R_{\mu}^{\prime}$ which is a contradiction. Suppose $c^{e} \in R_{\mu}$, then $c^{e}=x+n x_{\mu}+m\left(c^{0}-T_{\mu} x_{\mu}\right)$ for some $x \in R_{\mu}^{\prime}$ and some integers $n$ and $m$, but $x_{\mu}$ and $T_{\mu} x_{\mu} \in C[p]^{0}$, so $c^{e}=x \in R_{\mu}^{\prime}$ which 
is also a contradiction. Suppose $\measuredangle\left(T_{\mu}\right)$ has property $2^{\circ}$, then we can find $x_{\mu} \in L_{4}\left(T_{\mu}\right)$ such that $\left(R_{\mu}^{\prime}+\left[c^{0}\right]+\left[c^{e}\right]\right) \cap\left(\left[x_{\mu}\right] \oplus\left[T_{\mu} x_{\mu}-q x_{\mu}\right]\right)=\{0\}$. Let $R_{\mu}=R_{\mu}^{\prime}+\left[x_{\mu}\right]+\left[c^{e}-T_{\mu} x_{\mu}+q x_{\mu}\right]$, then clearly $R_{\mu}$ satisfies above (a), (b) and (c). And $c^{0}$ and $c^{e} \notin R_{\mu}$ also holds. Suppose $c^{0} \in R_{\mu}$, then $c^{0}=x+n x_{\mu}+m\left(c^{e}-T_{\mu} x_{\mu}+q x_{\mu}\right)$ for some $x \in R_{\mu}^{\prime}$ and some integers $n$ and $m$, but $x_{\mu} \in C[p]^{0}$ and $T_{\mu} x_{\mu}-q x_{\mu} \in C[p]^{e}$, hence we have $c^{0}=x^{0}+n x_{\mu}$, that is, $-x^{0}+c^{0}=n x_{\mu}$. Our choice of $x_{\mu}$ implies $n x_{\mu}=0=-x^{0}+c^{0}$, so we have $c^{0}=x^{0} \in R_{\mu}^{\prime}$ which is a contradiction. Suppose $c^{e} \in S_{\mu}$, then $c^{e}=x+n x_{\mu}+m\left(c^{e}-T_{\mu} x_{\mu}+q x_{\mu}\right)$ for some $x \in R_{\mu}^{\prime}$ and some integers $n$ and $m$. Hence $-x+(1-m) c^{e}=n x_{\mu}-m\left(T_{\mu} x_{\mu}-q x_{\mu}\right)$, but by our choice of $x_{\mu}$ we see $-x+(1-m) c^{e}=0=n x_{\mu}-m\left(T_{\mu} x_{\mu}-q x_{\mu}\right)$. This implies $n=m=0 \bmod p$, so $c^{e}=x \in R_{\mu}^{\prime}$ which is also a contradiction. Suppose $\Delta\left(T_{\mu}\right)$ has property $3^{\circ}$, then we can find $x_{\mu} \in \Delta\left(T_{\mu}\right)$ such that $\left(R_{\mu}^{\prime}+\left[c^{0}\right]+\left[c^{e}\right]\right) \cap\left(\left[x_{\mu}\right] \oplus\left[\left(T_{\mu} x_{\mu}\right)^{0}\right] \oplus\left[\left(T_{\mu} x_{\mu}\right)^{e}\right]\right)=\{0\}$. Let

$$
R_{\mu}=R_{\mu}^{\prime}+\left[x_{\mu}\right]+\left[c^{0}-\left(T_{\mu} x_{\mu}\right)^{0}\right]+\left[c^{e}-\left(T_{\mu} x_{\mu}\right)^{e}\right] .
$$

Then $R_{\mu}$ clearly satisfies (a), (b) and (c). And $c^{0}$ and $c^{e} \notin R_{\mu}$ can be seen as follows. Suppose $c^{0}=x+n x_{\mu}+m\left(c^{0}-\left(T_{\mu} x_{\mu}\right)^{0}\right)+m^{\prime}\left(c^{e}-\left(T_{\mu} x_{\mu}\right)^{e}\right)$ for some $x \in R_{\mu}^{\prime}$ and integers $n, m$ and $m^{\prime}$, then

$$
c^{0}=x^{0}+n x_{\mu}+m\left(c^{0}-\left(T_{\mu} x_{\mu}\right)^{0}\right),
$$

so $-x^{0}+(1-m) c^{0}=n x_{\mu}-m\left(T_{\mu} x_{\mu}\right)^{0}$. This implies $n x_{\mu}-m\left(T_{\mu} x_{\mu}\right)^{0}=$ $0=-x^{0}+(1-m) c^{0}$ by our choice of $x_{\mu}$. Hence $m=0$ and $c^{0}=x^{0} \in R_{\mu}^{\prime}$ which is a contradiction. We can see also $c^{e} \notin R_{n}$ for same reason. And $x_{\mu}$ and $c-T_{\mu} x_{\mu} \in R_{\mu}$ is clear. The construction of $R_{\mu}$ for $\left\langle\left(T_{\mu}\right)\right.$ having one of properties $1^{e} \sim 3^{e}$ is exactly similar by exchanging odd for even.

Let $Q=\bigcup_{\lambda \in A} R_{\lambda}$. Then the above properties (a) $\sim(\mathrm{d})$ for all $R_{\lambda}$ guarantee that $Q$ is a subgroup between $\Sigma[p]$ and $C[p]$ not invariant under any $T_{\lambda}(\lambda \in \Lambda)$ but invariant under $P_{e}$.

\section{REFERENCES}

1. G. Baumslag, Hopficity and abelian groups, Topics in abelian groups, Proceedings of the New Mexico Symposium on Abelian Groups, 1962, 331-335.

2. R. A. Beaumont and R. S. Pierce, Partly transitive modules and monules with proper isomorphic submodules, Trans. Amer. Math. Soc. 91 (1959) 209-219.

3. P. Crawley, An infinite primary abelian group without proper isomorphic subgroups, Bull. Amer. Math. Soc. 68 (1962) 463-467.

4. L. Fuchs, Abelian groups, Pergamon Press, Oxford, 1960.

5. E. Hewitt and K. A. Ross. Abstract harmonic analysis I, Academic Press, New York, 1963.

6. J. M. Irwin, F. Richman and E. A. Walker, Countable direct sums of torsion complete groups, Proc. Amer. Math. Soc. 17 (1966), 763-766. 
7. R. S. Pierce, The endomorphism rings of primary abelian groups, Topics in abelian groups, Proceedings of the New Mexico Symposium on Abelian Groups, (1962) 215-310.

Received August 22, 1967.

WAyne State University and

HOKKAIDO UNIVERSITY 



\section{Pacific Journal of Mathematics \\ Vol. 29, No. 1 \\ May, 1969}

Jorge Alvarez de Araya, A Radon-Nikodým theorem for vector and operator

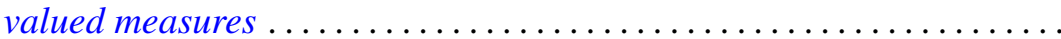

Deane Eugene Arganbright, The power-commutator structure of finite

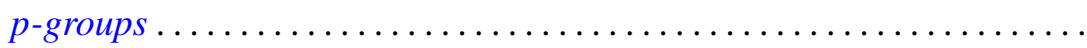

Richard Eugene Barlow, Albert W. Marshall and Frank Proschan, Some

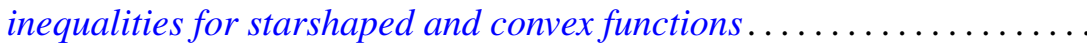

David Clarence Barnes, Some isoperimetric inequalities for the eigenvalues

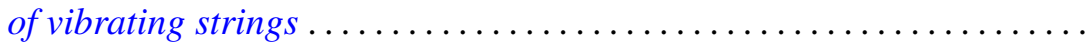

David Hilding Carlson, Critical points on rim-compact spaces ...........

Allan Matlock Weber Carstens, The lattice of pretopologies on an arbitrary

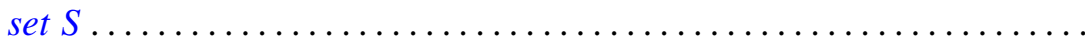

S. K. Chatterjea, A bilateral generating function for the ultraspherical

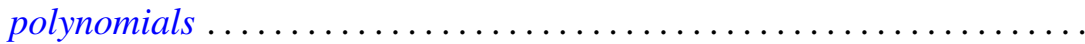

Ronald J. Ensey, Primary Abelian groups modulo finite groups ......... 77

Harley M. Flanders, Relations on minimal hypersurfaces ............ 83

Allen Roy Freedman, On asymptotic density in n-dimensions........... 95

Kent Ralph Fuller, On indecomposable injectives over artinian rings...... 115

George Isaac Glauberman, Normalizers of p-subgroups in finite groups . . . 137

William James Heinzer, On Krull overrings of an affine ring ........... 145

John McCormick Irwin and Takashi Ito, A quasi-decomposable abelian group without proper isomorphic quotient groups and proper isomorphic subgroups.

Allan Morton Krall, Boundary value problems with interior point boundary conditions

John S. Lowndes, Triple series equations involving Laguerre

polynomials

Philip Olin, Indefinability in the arithmetic isolic integers

Ki-Choul Oum, Bounds for the number of deficient values of entire functions whose zeros have angular densities..

R. D. Schafer, Standard algebras ....................

Wolfgang M. Schmidt, Irregularities of distribution. III.

Richard Alfred Tapia, An application of a Newton-like method to the Euler-Lagrange equation 